\title{
Descriptor Differential Scale
}

National Cancer Institute

\section{Source}

National Cancer Institute. Descriptor Differential Scale. NCI Thesaurus. Code C21139.

Measures the sensory and affective components of pain using a magnitude ratio scale.

(Gracey 1988) 